\title{
Comparison of Clinical and Anatomical Outcomes between Delaminated Rotator Cuff Tear and Single Layer Rotator Cuff Tear
}

\author{
Jin-Woo Park, Sung-Hoon Moon ${ }^{\varpi}$, Jun-Hee Lee \\ Department of Orthopedic Surgery, Kangwon National University School of Medicine, Chuncheon, Korea
}

\begin{abstract}
Background: Delaminated rotator cuff tear is known to be a degenerative tear having a negative prognostic effect. This study undertook to compare the anatomical and clinical outcomes of delaminated tears and single layer tears.

Methods: Totally, 175 patients with medium to large rotator cuff tears enrolled for the study were divided into 2 groups, based on the tear pathology: single layer tear (group 1) and delaminated tear (group 2). Preoperatively, length of the remnant tendon, muscle atrophy of supraspinatus (SS), and fatty degeneration of SS and infraspinatus (IS) muscles were assessed on magnetic resonance imaging (MRI). For follow-up, the repair integrity of the rotator cuff was evaluated by ultrasonography. Clinical outcomes were assessed by evaluating the Constant score (CS) and Korean Shoulder Score (KSS).

Results: Retears were detected in 6 cases of group 1 (6.5\%) and 11 cases of group 2 (13.3\%). Although higher in group 2, the retear rate was significantly not different $(p=0.133)$. Preoperative MRI revealed length of remnant tendon to be $15.46 \pm 3.60 \mathrm{~mm}$ and $14.17 \pm 3.16$ $\mathrm{mm}(p=0.013)$, and muscle atrophy of SS (occupation ratio) was $60.54 \pm 13.15$ and $56.55 \pm 12.88$ ( $p=0.045$ ), in group 1 and group 2 , respectively. Fatty degeneration of SS and IS in both groups had no significant differences. Postoperatively, no significant differences were observed for CS and KSS values between the groups.

Conclusions: Delaminated rotator cuff tears showed shorter remnant tendon length and higher muscle atrophy that correlate to a negative prognosis. These prognostic effects should be considered during delaminated rotator cuff tear treatment.
\end{abstract}

(Clin Shoulder Elbow 2018;21(4):207-212)

Key Words: Shoulder; Rotator cuff tears; Delaminated tear; Whole layer repair; Retear

\section{Introduction}

Delamination of the rotator cuff is commonly found in rotator cuff tears, with a higher incidence seen in medium sized tears. ${ }^{1,2}$ The incidence of delamination is reported to be between $38 \%$ to $92 \%{ }^{1-3)}$ However, the exact etiology of delamination is not proven. Some authors have reported the anatomical characteristics of the rotator cuff, and demonstrated that delamination occurs between the two layers. ${ }^{4,5}$ Several authors have reported that delamination is a negative prognostic factor in clinical and anatomical outcomes after rotator cuff repair. ${ }^{1,2,6)}$ However, a controversy still exists since some authors suggest that delamination has no effect on the outcomes of rotator cuff repair.?
This study was therefore undertaken to assess the clinical and anatomical outcomes after rotator cuff repair by comparing delaminated rotator and single layer rotator cuff tears.

\section{Methods}

This study was designed as a retrospective comparative study (level III).

\section{Patient Selection}

Between March 2012 to March 2016, 175 patients who had undergone arthroscopic repair for medium to large rotator cuff tears and had followed-up for at least 1 year, were enrolled for

Received September 9, 2018. Revised October 15, 2018. Accepted October 16, 2018.

Correspondence to: Sung-Hoon Moon

Department of Orthopedic Surgery, Kangwon National University School of Medicine, 1 Gangwondaehak-gil, Chuncheon 24341, Korea Tel: +82-33-258-9224, Fax: +82-33-258-2149, E-mail: luteus@naver.com, ORCID: https://orcid.org/0000-0003-4728-842X

IRB approval: Kangwon National University Hospital (KNUH-2017-09-011-003).

Financial support: None. Conflict of interests: None. 

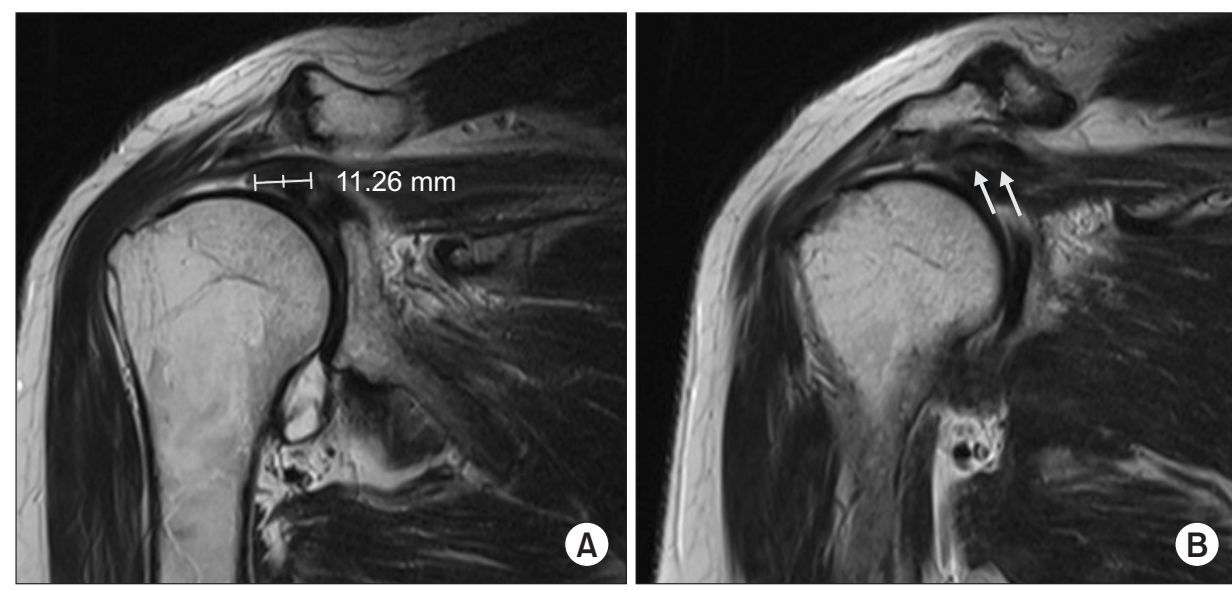

Fig. 1. Measurement of the remnant tendon length and evaluation of delamination was done on oblique coronal $\mathrm{T} 2$ weighted image in preoperative magnetic resonance imaging. (A) Remnant tendon length was measured from the midpoint of lateral edge of the torn tendon to the musculotendinous junction on the clearest image obtained of the 'midportion of tendon'. (B) Delamination of tendon was evaluated on oblique coronal image (arrows).

the study. Cases were divided into two groups: patients with single layer rotator cuff tear (group 1) and patients with delaminated rotator cuff tear (group 2).

The exclusion criteria were: (1) massive cuff tear, (2) irreparable tear, (3) glenohumeral arthritis, (4) stiff shoulder, (5) revision of rotator cuff tear, and (6) previous surgery on ipsilateral shoulder.

\section{Preoperative Evaluation}

All patients were subjected to preoperative magnetic resonance imaging (MRI), and interpretation and measurement of the obtained oblique coronal images was performed by a single surgeon to assess delamination of the tendon and length of the remnant tendon (Fig. 1). ${ }^{8}$ The clearest image of the tendon midportion was used to assess the remnant tendon length, which was measured from midpoint of the lateral edge of the torn tendon to the musculotendinous junction. The exact remnant tendon length was difficult to determine in delaminated tears due to the difference in length between the outer and inner layer tendons. Therefore, we measured the tendon length on images that showed minimal difference between the two layers, and determined remnant tendon length as the average of two tendon lengths. Muscle atrophy of the supraspinatus (SS) was measured by using the occupation ratio on oblique sagittal ' $Y$ ' view (Fig. 2). The occupation ratio is defined as the ratio between the crosssectional area of the SS muscle and SS fossa. It was obtained using a picture archiving and communication system, as described by Thomazeau et al. ${ }^{9)}$ Fatty degeneration of SS and infraspinatus (IS) were also measured using the grading system developed by Goutallier et al. (Fig. 3). ${ }^{10)}$

The Constant Score (CS) and Korean Shoulder Score (KSS) were evaluated by questionnaires before surgery.

\section{Surgical Procedure}

All patients were subjected to arthroscopic rotator cuff repair in the beach chair position. Conventional glenohumeral exami-

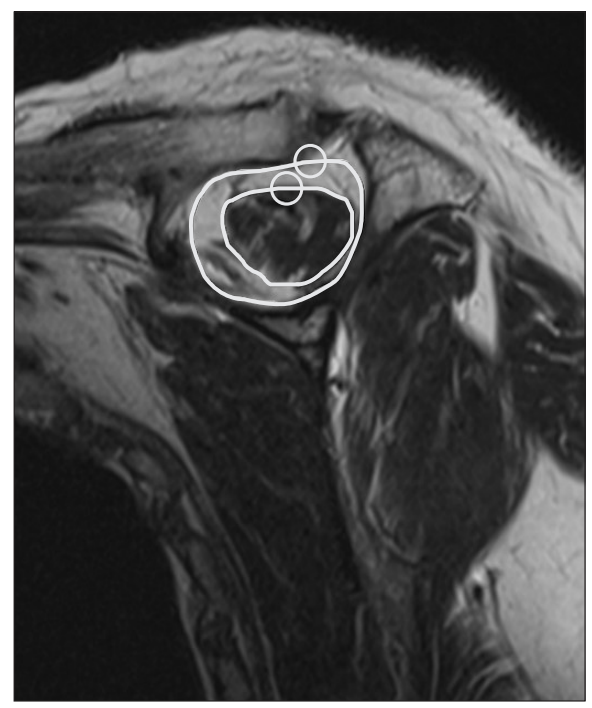

Fig. 2. Muscle atrophy of supraspinatus was measured using occupation ratio at ' $\mathrm{Y}$ ' view on oblique sagittal image in preoperative magnetic resonance imaging.

nation was done using the standard posterior and anterior portal. Tear shape and delamination were evaluated on bursoscopy using the lateral portal. The location of delamination and direction of retracted inner-layer were measured if delamination was detected (Fig. 4).

Suture bridge double row (SBDR) repair was performed in cases of single layer rotator cuff tear, whereas SBDR (En-masse SBDR, EM-SBDR) repair was done in all cases of delaminated rotator cuff tear (Fig. 5). ${ }^{7)}$ During EM-SBDR, synovial tissue between the two layers and the non-viable tissues were debrided first, after which the more retracted inner layer was secured with a tension suture; the suture was passed through the whole tendon whilst maintaining tension of the tension suture. After passing all sutures through the whole tendon, a conventional suture bridge repair was performed. 

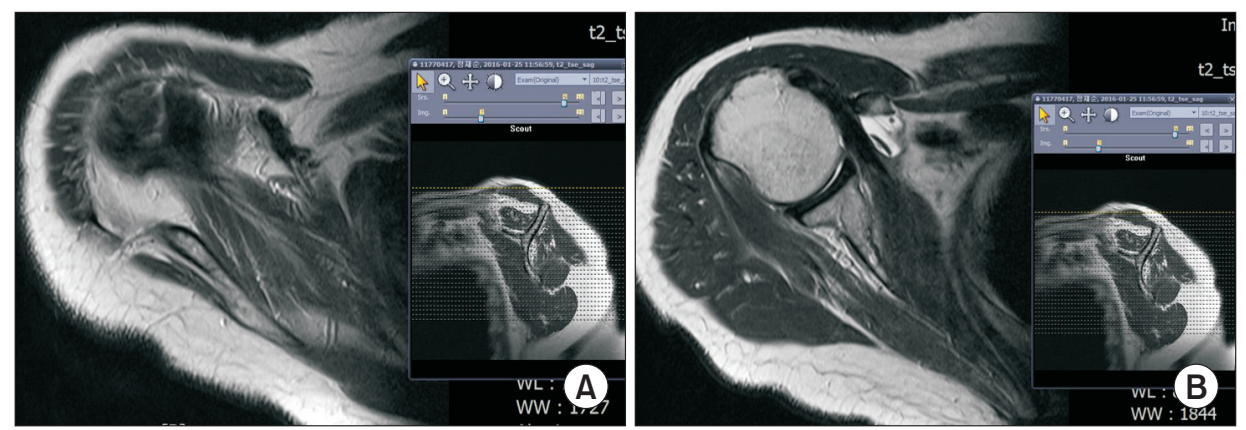

Fig. 3. Fatty degeneration were evaluated on axial and oblique sagittal images in the preoperative magnetic resonance imaging. (A) Supraspinatus. (B) Infraspinatus.
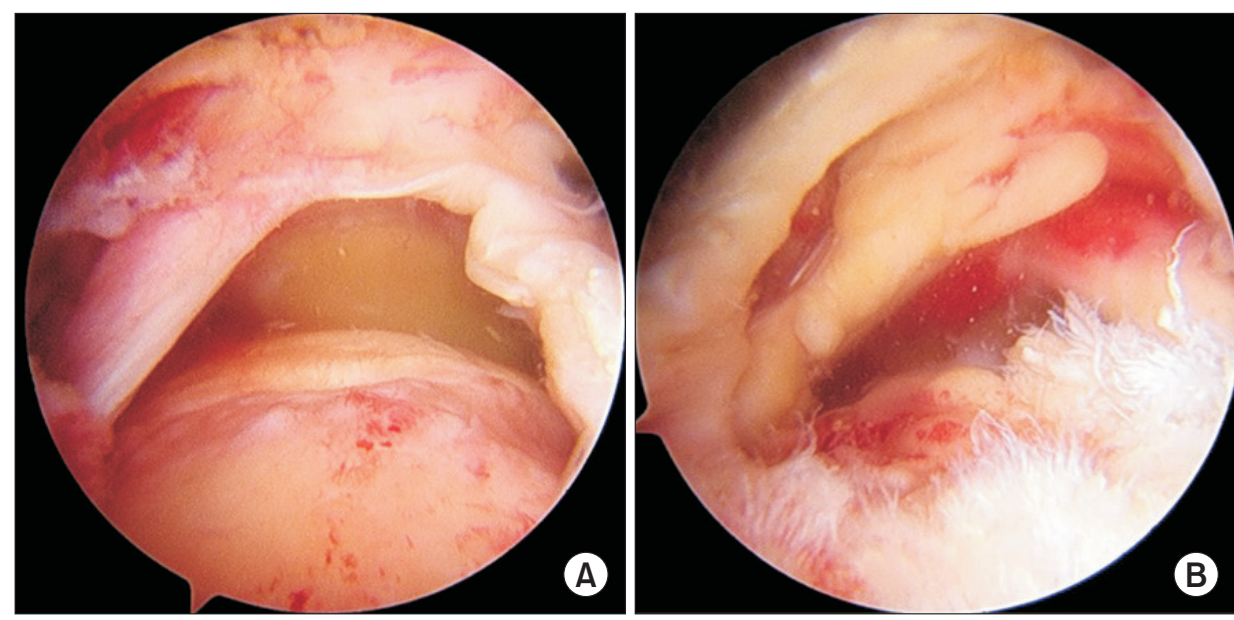

Fig. 4. Intraoperatively, the tear shape was evaluated at the lateral portal of bursoscopy. (A) Single layer tear. (B) Delaminated tear.
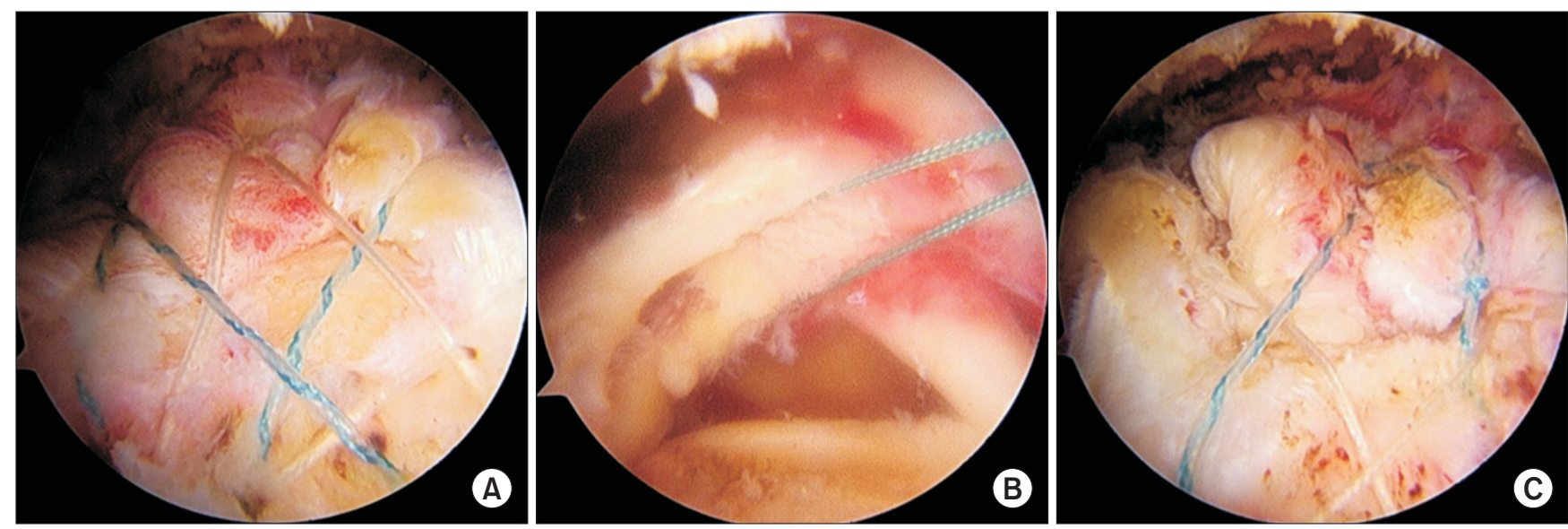

Fig. 5. Rotator cuff repair. (A) Suture bridge double row repair was done for single layer tears. (B, C) En-masse suture bridge double row repair was done in cases of delaminated tears.

\section{Postoperative Rehabilitation}

Postoperative rehabilitation was the same for both groups. Arm abduction brace was applied for 6 weeks after surgery. After the first 3 weeks, pendulum exercises and passive forward elevation to $90^{\circ}$ was initiated. At the 4th week, unlimited passive range of motion exercises were started. At 6th week after surgery, the brace was removed, and active assisted range of motion exercises were started, followed by initiation of strengthening exercise at 3 months postoperatively.

\section{Postoperative Evaluation}

During follow-up, repair integrity was evaluated by ultrasonography at 3, 6, 12, and 24 months postoperatively. Retear was defined as discontinuity of tendon and graded more than 4 in the Sugaya grading system (Fig. 6). ${ }^{11}$

CS and KSS were assessed at the final follow-up for overall clinical outcomes. 

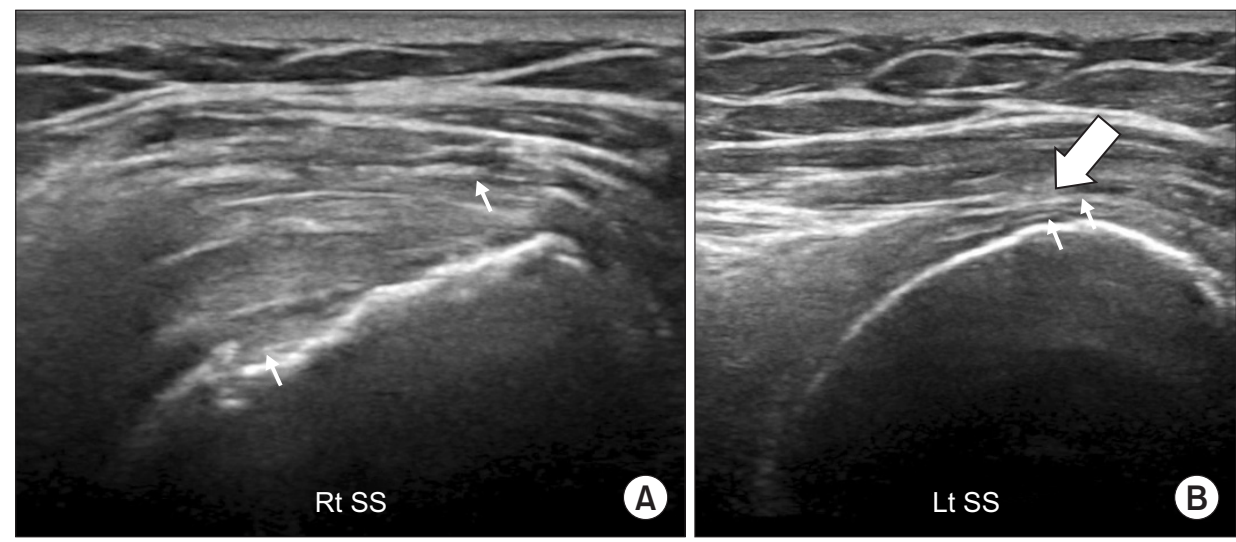

Fig. 6. Repair integrity was followed by ultrasonography. (A) Intact repair integrity. (B) Retear. (A) Arrows indicate intact articular and bursal layer of repaired tendon. (B) Arrow indicates discontinuity of tendon. Rt SS: supraspinatus, Lt SS: supraspinatus.

Table 1. Comparison of Retear Rate between Single Layer Tear and Delaminated Tear

\begin{tabular}{cccc}
\hline & Group 1 $(\mathrm{n}=92)$ & Group 2 $(\mathrm{n}=83)$ & $p$-value \\
\hline Retear & $6(6.5)$ & $11(13.3)$ & 0.133 \\
\hline
\end{tabular}

Values are presented as number (\%).

Group 1: single layer rotator cuff tear, Group 2: delaminated rotator cuff tear.

${ }^{*}$ Retear rate was not significantly different between two groups.

\section{Statistical Analysis}

Student t-test was used for comparing muscle atrophy, fatty degeneration (of SS and IS), age, and preoperative and postoperative clinical scores between the two groups. Retear rate was compared between both groups using the chi-square test. Differences of remnant tendon length, muscle atrophy, and fatty degeneration between retear and non-retear groups were analyzed using the Mann-Whitney U-test. The critical value for significance was set as $p<0.05$. Statistical analyses were performed with IBM SPSS statistical package ver. 19.0 (IBM Co., Armonk, NY, USA).

\section{Results}

Of the 175 patients enrolled, 92 were designated to group 1, and 83 to group 2 . The mean follow-up period was $15.9 \pm 4.8$ months (range, 12-27 months).

Retears were detected in 6 cases (6.5\%) of group 1 and 11 cases (13.3\%) of group 2. Although the retear rate was higher in group 2 , there was no significant difference $(p=0.133)$ (Table 1$)$. In group 1, retear appeared at postoperative 3 months in 2 cases and 6 months in 4 cases. In group 2, retear appeared at postoperative 3 months in 6 cases and 6 months in 5 cases.

In preoperative MRI, length of the remnant tendon was $15.46 \pm 3.60 \mathrm{~mm}$ in group 1 , and $14.17 \pm 3.16 \mathrm{~mm}$ in group $2(p=0.013)$, and muscle atrophy of SS (occupation ratio) was $60.54 \pm 13.15$ in group 1 , and $56.55 \pm 12.88$ in group 2 $(p=0.045)$. Fatty degeneration of SS and IS showed no significant differences (SS: $1.55 \pm 0.65$ and $1.57 \pm 0.55$ in group 1 and
Table 2. Comparison of Factors between Two Groups

\begin{tabular}{lccc}
\hline \multicolumn{1}{c}{ Factor } & Group 1 & Group 2 & p-value \\
\hline Age (yr) & $64.32 \pm 9.32$ & $64.45 \pm 8.79$ & 0.924 \\
\hline Remnant tendon $(\mathrm{mm})$ & $15.46 \pm 3.60$ & $14.17 \pm 3.16$ & $0.013^{\dagger}$ \\
Muscle atrophy (\%) & $60.54 \pm 13.15$ & $56.55 \pm 12.88$ & $0.045^{\dagger}$ \\
$\begin{array}{c}\text { Fatty degeneration } \\
\text { (supraspinatus) }\end{array}$ & $1.55 \pm 0.65$ & $1.57 \pm 0.55$ & 0.896 \\
$\begin{array}{c}\text { Fatty degeneration } \\
\text { (infraspinatus) }\end{array}$ & $1.71 \pm 0.64$ & $1.86 \pm 0.65$ & 0.127 \\
\hline
\end{tabular}

Values are presented as mean \pm standard deviation.

Group 1: single layer rotator cuff tear, Group 2: delaminated rotator cuff tear.

${ }^{\star}$ Muscle atrophy was presented as occupation ratio (\%) of supraspinatus muscle.

${ }^{\dagger}$ Remnant tendon length and muscle atrophy were significantly different.

2 , respectively, $p=0.896 ;$ IS: $1.71 \pm 0.64$ and $1.86 \pm 0.65$ in group 1 and 2, respectively, $p=0.127$ ) (Table 2).

When comparing between groups based on retear, the remnant tendon length and fatty degeneration of IS were significantly different between the retear and non-retear groups (remnant tendon length: $12.90 \pm 3.67$ and $15.05 \pm 3.37$ in retear and non-retear group, respectively, $p=0.012$; fatty degeneration of IS: $2.18 \pm 0.64$ and $1.73 \pm 0.63$ in retear and non-retear group, respectively, $p=0.008$ ) (Table 3 ).

At final follow-up, clinical scores were improved in all cases. CS were $71.64 \pm 8.09$ in group 1 and $71.14 \pm 7.05$ in group 2 $(p=0.667)$. KSS were $75.51 \pm 8.64$ in group 1 and $74.65 \pm 8.58$ in group $2(p=0.510)$. There were no significant differences in clinical scores between both groups (Table 4).

\section{Discussion}

Results from our study showed no significant differences in the retear rates between single layer tear and delaminated tear groups, indicating that delamination is not related to anatomical outcomes.

Although a known degenerative change of rotator cuff tears, 
Table 3. Comparison of Factors between Retear and Non-retear Groups

\begin{tabular}{lccc}
\multicolumn{1}{c}{ Factor } & Retear & Non-retear & $p$-value \\
\hline Age (yr) & $66.59 \pm 8.32$ & $64.14 \pm 9.12$ & 0.285 \\
\hline Remnant tendon $(\mathrm{mm})$ & $12.90 \pm 3.67$ & $15.05 \pm 3.37$ & $0.012^{\dagger}$ \\
Muscle atrophy (\%) & $53.88 \pm 11.29$ & $59.16 \pm 13.25$ & 0.064 \\
$\begin{array}{c}\text { Fatty degeneration } \\
\text { (supraspinatus) }\end{array}$ & $1.82 \pm 0.64$ & $1.53 \pm 0.59$ & 0.061 \\
$\begin{array}{c}\text { Fatty degeneration } \\
\text { (infraspinatus) }\end{array}$ & $2.18 \pm 0.64$ & $1.73 \pm 0.63$ & $0.008^{\dagger}$ \\
\hline
\end{tabular}

Values are presented as mean \pm standard deviation.

${ }^{*}$ Muscle atrophy was presented as occupation ratio (\%) of supraspinatus muscle.

${ }^{\dagger}$ Remnant tendon length and fatty degeneration of infraspinatus were significantly different.

the cause of delamination is not clearly understood. Sonnabend et al. ${ }^{4)}$ reported delamination to be generally found between layers having different fiber orientation, with local ischemia contributing to delamination. Furthermore, delamination revealed the presence of a synovial lining, which was found to be related to symptom duration. Hence, the authors recommended debridement of the synovial lining during rotator cuff repair.

Some authors report that delamination is a poor prognostic factor for rotator cuff repair. ${ }^{1,2)}$ However, recent studies have reported no significant differences in the clinical outcomes when comparing delamination with single layer tear. ${ }^{3,7,12,13)}$

Kim et al. ${ }^{14)}$ compared the clinical and anatomical outcomes of delaminated and non-delaminated groups, and reported no significant differences between both groups. In our study, good clinical outcomes (CS, KSS) were achieved across all cases, with no significant differences between single layer tears and delaminated tears.

Considering tendon healing, Park et al. ${ }^{7}$ reported no significant association between delamination and retear rate. Kim et al. ${ }^{14)}$ classified three types of tendon healing: complete healing, partially healed defect, and retear. They reported partially healed defects were significantly high in delaminated tears but had a lower rate in retears, thereby indicating the cause to be differing tension between the layers after repair. Their study concluded that delamination may not be a poor prognostic factor for rotator cuff repair. In our study, the follow-up ultrasonography of retears determined that although not significant, the retear rate was higher in delaminated tears.

Several researches have studied the predictive factors responsible for retear after rotator cuff repair. ${ }^{15-17)}$ Kim et al. ${ }^{18)}$ evaluated factors affecting healing after rotator cuff repair, and reported that multiple factors influence tendon healing, including delamination and remnant tendon length. Additionally, they suggested the extent of retraction and occupation ratio as determining prognostic factors. Jeong et al. ${ }^{19)}$ reported the cutoff values of occupation ratio of SS and fatty infiltration of the IS were $43 \%$
Table 4. Clinical Outcomes at Final Follow-up between Single Layer Tear and Delaminated Tear

\begin{tabular}{lccc}
\hline & Group 1 & Group 2 & $p$-value \\
\hline Constatnt score & $71.64 \pm 8.09$ & $71.14 \pm 7.05$ & 0.667 \\
Korean Shoulder Score & $75.51 \pm 8.64$ & $74.65 \pm 8.58$ & 0.510 \\
\hline
\end{tabular}

Group 1: single layer rotator cuff tear, Group 2: delaminated rotator cuff tear.

and grade 2, respectively, which suggests these factors as strong predictors of retear. In our current study, in terms of existence of retear, remnant tendon length, and fatty degeneration of IS were significantly different between the retear and non-retear groups; the retear group showed significantly shorter remnant tendon length and higher fatty degeneration of IS compared to non-retear group. We therefore propose these to be predictable factors for higher retear rates.

The remnanat tendon length is also reported as a factor affecting tendon healing. ${ }^{8,20}$ Kim et al. ${ }^{8)}$ reported higher retear rate in patients with short remnant tendon, and suggested single row repair with low repair tension for such cases. In our study, the delaminated tear group showed significantly shorter remnant tendon length and higher muscle atrophy (lower occupation ratio) compared to the single layer tear group; however, these observations are unable to explain delamination as a negative prognostic factor for clinical outcomes.

There are few limitations in our study. First, this study was a retrospective study. Second, there was some difficulty in procuring exact preoperative MRI evaluation for remnant tendon length.

\section{Conclusion}

The data generated from the current study indicates that the retear rate is significantly not different between single layer tears and delaminated tears. Furthermore, there were no significant differences in clinical scores. Hence, it is reasonable to elucidate that delamination of the tendon is not a significant negative prognostic factor.

\section{References}

1. Boileau P, Brassart N, Watkinson DJ, Carles M, Hatzidakis AM, Krishnan SG. Arthroscopic repair of full-thickness tears of the supraspinatus: does the tendon really heal? J Bone Joint Surg Am. 2005;87(6):1229-40.

2. Flurin $\mathrm{PH}$, Landreau $\mathrm{P}$, Gregory $\mathrm{T}$, et al. Arthroscopic repair of full-thickness cuff tears: a multicentric retrospective study of 576 cases with anatomical assessment. Rev Chir Orthop Reparatrice Appar Mot. 2005;91(S8):31-42.

3. Sonnabend DH, Watson EM. Structural factors affecting the outcome of rotator cuff repair. J Shoulder Elbow Surg. 2002; 
11(3):212-8.

4. Sonnabend DH, Yu Y, Howlett CR, Harper GD, Walsh WR. Laminated tears of the human rotator cuff: a histologic and immunochemical study. J Shoulder Elbow Surg. 2001;10:10915.

5. Mochizuki T, Sugaya H, Uomizu M, et al. Humeral insertion of the supraspinatus and infraspinatus. New anatomical findings regarding the footprint of the rotator cuff. Surgical technique. J Bone Joint Surg Am. 2009;91 Suppl 2 Pt 1:1-7.

6. Mori D, Funakoshi N, Yamashita F. Arthroscopic lamina-specific double-row fixation for large delaminated rotator cuff tears. Arthrosc Tech. 2014;3(6):e667-71.

7. Park JY, Lhee SH, Oh KS, Moon SG, Hwang JT. Clinical and ultrasonographic outcomes of arthroscopic suture bridge repair for massive rotator cuff tear. Arthroscopy. 2013;29(2):280-9.

8. Kim YK, Moon SH, Cho SH. Treatment outcomes of singleversus double-row repair for larger than medium-sized rotator cuff tears: the effect of preoperative remnant tendon length. Am J Sports Med. 2013;41(10):2270-7.

9. Thomazeau H, Rolland Y, Lucas C, Duval JM, Langlais F. Atrophy of the supraspinatus belly. assessment by MRI in 55 patients with rotator cuff pathology. Acta Orthop Scand. 1996; 67(3):264-8.

10. Goutallier D, Postel JM, Gleyze P, Leguilloux P, Van Driessche S. Influence of cuff muscle fatty degeneration on anatomic and functional outcomes after simple suture of full-thickness tears. J Shoulder Elbow Surg. 2003;12(6):550-4.

11. Sugaya H, Maeda K, Matsuki K, Moriishi J. Repair integrity and functional outcome after arthroscopic double-row rotator cuff repair. A prospective outcome study. J Bone Joint Surg Am. 2007;89(5):953-60.

12. Kim SJ, Choi YR, Lee HH, Chun YM. Surgical results of delaminated rotator cuff repair using suture-bridge technique with all-layers or bursal layer-only repair. Am J Sports Med. 2016;44(2):468-73.

13. MacDougal GA, Todhunter CR. Delamination tearing of the rotator cuff: prospective analysis of the influence of delamination tearing on the outcome of arthroscopically assisted mini open rotator cuff repair. J Shoulder Elbow Surg. 2010;19(7): 1063-9.

14. Kim CW, Kim JH, Gwak HC, Park JH. The comparison of outcomes between delaminated and nondelaminated rotator cuff tear repair: is delamination a negative prognostic factor? J Shoulder Elbow Surg. 2017;26(2):216-24.

15. Khair MM, Lehman J, Tsouris N, Gulotta LV. A systematic review of preoperative fatty infiltration and rotator cuff outcomes. HSS J. 2016;12(2):170-6.

16. Le $B T, W u X L$, Lam $P H$, Murrell GA. Factors predicting rotator cuff retears: an analysis of 1000 consecutive rotator cuff repairs. Am J Sports Med. 2014;42(5):1134-42.

17. McElvany MD, McGoldrick E, Gee AO, Neradilek MB, Matsen FA 3rd. Rotator cuff repair: published evidence on factors associated with repair integrity and clinical outcome. Am J Sports Med. 2015;43(2):491-500.

18. Kim YK, Jung KH, Kim JW, Kim US, Hwang DH. Factors affecting rotator cuff integrity after arthroscopic repair for mediumsized or larger cuff tears: a retrospective cohort study. J Shoulder Elbow Surg. 2018;27(6):1012-20.

19. Jeong HY, Kim HJ, Jeon YS, Rhee YG. Factors predictive of healing in large rotator cuff tears: is it possible to predict retear preoperatively? Am J Sports Med. 2018;46(7):1693-700.

20. Tashjian RZ, Hung M, Burks RT, Greis PE. Influence of preoperative musculotendinous junction position on rotator cuff healing using single-row technique. Arthroscopy. 2013; 29(11):1748-54. 